\title{
Geometric interpretation of the dark energy from projected hyperconical universes
}

\author{
R. Monjo* \\ Department of Algebra, Geometry and Topology, Faculty of Mathematics, \\ Complutense University of Madrid, 28040 Madrid, Spain
}

(Received 10 May 2018; published 7 August 2018)

\begin{abstract}
This paper explores the derivation of dark energy from a locally conformal projection of hyperconical universes. It focuses on the analysis of theoretical compatibility between the intrinsic view of the standard cosmology and an adequate transformation of hyperconical manifolds. Choosing some parametric family of locally conformal transformations and taking regional (second order) equality between the Hubble parameter of both theories, it is predicted that the dark energy density is $\Omega_{\Lambda}=0.6937181(2)$. In particular, we used a radially distorted stereographic projection, the distortion parameter of which is theoretically predicted about $\alpha=0.325 \pm 0.005$ and empirically fitted as $\alpha=0.36 \pm 0.02\left(\chi_{0}^{2}=562\right)$ according to $580 \mathrm{SNe}$ Ia observations.
\end{abstract}

DOI: 10.1103/PhysRevD.98.043508

\section{INTRODUCTION}

\section{A. Motivation}

Astronomical observations suggest that the universe is spatially flat, accelerating, and composed of predominately dark energy and dark matter. However, despite of the great agreement of the standard cosmology with this, the nature of dark energy still remains an open issue. In fact, it is not measured from model-independent observations. Moreover, observations of the universe's flatness are also based on the validity assumption of the current model. Changing the frame theory, the same observations can be explained using another geometry, even with positive curvature [1].

Recently, geometrical interpretations for the dark energy have been explored [2-4]. For instance, conformal gravity can be used to obtain both dark energy and matter [2]. The hypothesis of the conformal cosmology is based on the invariance of the geometry under any local conformal transformation [3]. Alternatively, Maia et al. [4] obtained dark energy as warp in the universe given by the extrinsic curvature within a Friedmann-Robertson-Walker (FRW) universe embedded into a five-dimensional constant curvature bulk.

The FRW metric used in the standard $\Lambda$ CDM model is obtained from a static manifold with constant spatial curvature and an additional scale factor $a(t)$ to explain the expansion. However, other theories based on varying extrinsic geometries can explain the expansion $[5,6]$. For example, hyperconical universes produce inhomogeneous metrics compatible with the observed expansion and they locally approach to the flat FRW metric. To be consistent with the

*rmoncho@ucm.es
$\Lambda \mathrm{CDM}$ model, it can be assumed as a local perturbation theory in inhomogeneous universes expanding regardless of the matter content [7]. On the other hand, physical properties of inhomogeneous spaces are interesting because they can be used to trace dark energy and dark matter [8].

As a continuation of the study presented in [7], this letter explores an interpretation of the dark energy according to locally conformal projections of hyperconical universes. For this purpose, the analysis deeps in its theoretical compatibility with the $\Lambda \mathrm{CDM}$ model for both at low and high redshift regions, particularly including the first acoustic peak in the Cosmic Microwave Backward (CMB) radiation. Therefore, a quick review of the standard model is required to compare with the hyperconical model.

\section{B. The first $\mathrm{CMB}$ acoustic peak}

Hubble parameter. The Hubble parameter is defined as $H:=\dot{a} / a$. For the $\Lambda \mathrm{CDM}$ model, it is obtained from the second Friedmann equation according to the matter $\left(\Omega_{m}\right)$, radiation $\left(\Omega_{r}\right)$ and dark energy $\left(\Omega_{\Lambda}\right)$ contents. Assuming zero curvature $\left(\Omega_{K}=0\right)$, the Hubble parameter $H_{\Lambda}$ of the standard model is

$$
H_{\Lambda}=H_{o} \sqrt{\Omega_{r}(1+z)^{4}+\Omega_{m}(1+z)^{3}+\Omega_{\Lambda}} .
$$

Comoving distance. The physical (proper) radial distance or comoving distance, $\hat{r}^{\prime}$, is given by the null geodesic with no angular variations in the considered metric. This distance can be written using the redshift $z$ and the Hubble parameter, $H$, with the scale factor expressed as $a / a_{\hat{o}}=$ $1 /(1+z)$ where $a_{\hat{o}} \equiv 1$ is the current value of $a$. For the $\Lambda \mathrm{CDM}$ universes, one can find: 


$$
\hat{r}_{\Lambda}^{\prime}(z)=\sin _{K} \int_{0}^{z} \frac{d z}{H_{\Lambda}(z)}
$$

where $\sin _{K} x:=\lim _{\epsilon \rightarrow K} \epsilon^{-1 / 2} \sin \left(\epsilon^{1 / 2} x\right), K=-\Omega_{K} H_{o}^{2}$; i.e., $\sin _{0}(x)=x, \sin _{+1} x=\sin x$ and $\sin _{-1} x=\sinh x$.

Sound horizon. The first CMB peak in the multipole moment is given by the sound horizon $r_{S}$ at decoupling between baryons and photons. It can be obtained according to the integral of the effective sound speed $c_{s}(z)$ of the coupled baryon-photon plasma $[9,10]$,

$$
r_{S}(z)=\int_{0}^{t} c_{s}(t) a^{-1} d t=\int_{z}^{\infty} \frac{d z}{H(z)} c_{s}(z)
$$

The value of decoupling redshift $z_{\mathrm{dec}}$ is $1090.09 \pm 0.42$ according to the Planck mission [11]. The square value of $c_{s}(z)$ is:

$$
c_{s}^{2} \equiv \frac{\delta P_{\gamma}}{\delta \rho_{\gamma}+\delta \rho_{b}}=\frac{1}{3\left(1+R_{s}(z)\right)}
$$

where $\rho_{b}$ is the baryon density, $\rho_{\gamma}$ is the photon density and $R_{s}(z)$ is the baryon-to-photon density ratio, which can be expressed in terms of the $\mathrm{CMB}$ parameters as:

$$
R_{s}(z) \equiv \frac{3 \rho_{b}}{4} \frac{3 \omega_{b}}{\rho_{\gamma}} \frac{1}{4 \omega_{\gamma}} \frac{1}{1+z}
$$

where $\omega_{b}:=\Omega_{b} h^{2}$ and $\omega_{\gamma}:=\Omega_{\gamma} h^{2}=\Omega_{m} h^{2} /\left(1+z_{\mathrm{eq}}\right)$ are respectively the baryon and radiation contents, expressed with the reduced Hubble constant $h:=H /\left(100 \mathrm{~km} \mathrm{~s}^{-1} \mathrm{Mpc}^{-1}\right)$.

Therefore the sound horizon, i.e., the comoving distance traveled by a sound wave, is

$$
r_{S}\left(z_{\mathrm{dec}}\right)=\int_{z_{\mathrm{dec}}}^{\infty} \frac{d z}{H(z) \sqrt{3\left(1+R_{s}(z)\right)}}
$$

Then, the characteristic angle $\theta_{A}$ of the first CMB peak location and its corresponding multipole $\ell$ are related by:

$$
\ell=\frac{\pi}{\theta_{A}}=\pi \frac{r^{\prime}\left(z_{\mathrm{dec}}\right)}{r_{s}\left(z_{\mathrm{dec}}\right)}
$$

Empirically, the first CMB peak is found at $\ell=220.0 \pm$ 0.5 in agreement with the $\Lambda \mathrm{CDM}$ model $\left(\ell_{\Lambda}=220.9\right)$ [11].

Assuming the CMB measurements of the baryon and photon contents, $\omega_{b}:=\Omega_{b} h^{2}=0.02230(14)$ and $\omega_{\gamma}:=$ $\Omega_{\gamma} h^{2}=2.469(26) \times 10^{-5}[11]$, it is possible to estimate the baryon-to-photon density ratio $R_{S}(z)$ for any universe model according to Eq. (5). With this, the sound horizon $r_{S}$ and the multipole $\ell$ only depend on the theoretical Hubble parameter $H(z)$.

Therefore, the next sections are focused on the possible compatibility between the standard Hubble parameter and that obtained by the hyperconical universe for low and high redshifts. The local compatibility was found in [7], but for large distances it is required to apply some global projection to the hyperconical universe (Sec. III).

\section{HYPERCONICAL UNIVERSES}

Hypercones. Let $\mathrm{H}_{\beta \pm}^{4} \subset \mathbb{R}_{ \pm}^{5}:=\left(\mathbb{R}^{5} \backslash\{\mathbf{0}\}, \eta_{ \pm}\right)$, with metric $\eta_{ \pm}:=(+1,-1,-1,-1, \pm 1)$, be embedded hypercones with linear expansion and independent of the matter contents, i.e.,

$$
\mathrm{H}_{\beta \pm}^{4}:=\left\{(t, \vec{r}, u) \in \mathbb{R}_{ \pm}^{5}: t^{2}-\vec{r}^{2} \pm u^{2}=\beta_{ \pm}^{2} t^{2}\right\}
$$

with constant $\beta_{ \pm}^{2} \in\left\{\beta^{2} \in \mathbb{R}: \pm\left(\beta^{2}-1\right)>0\right\}$ and $\vec{r} \in \mathbb{R}^{3}$. Alternatively, it can be considered a Wick rotation for the coordinate $u$ with $\eta_{-}$and $\beta^{2}<1$. In any case, there must exists some diffeomorphism

$$
f:\left(\mathrm{H}_{\beta \pm}^{4}, \eta_{ \pm}\right) \rightarrow\left(\mathbb{R}_{>0} \times \mathbb{R}^{3}, g\right):=\mathbb{R}_{g}^{1,3}
$$

such that the metric $g$ inherits properties of $\mathrm{H}_{\beta \pm}^{4}$ and produces the same proper time in $\mathbb{R}_{g}^{1,3}$ as in the Minkowski spacetime.

Deformation. To preserve the proper time, the initial reference instant $t_{\hat{o}} \in \mathbb{R}_{\geq 0}$ is fixed by the observer although its actual position $(t, \vec{r}, u)$ is moving with time due to the expansion. That is, an expanding deformation $\mathcal{T}_{t}$ is applied to the spatial components $s:=(\vec{r}, u) \in \mathbb{R}^{4}$ of the observer according to:

$$
\mathcal{T}_{t}:\left(t_{\hat{o}}, s\right)=\left(t_{\hat{o}}, \frac{t}{t_{\hat{o}}} s\right) \in \mathbb{R}_{ \pm}^{5} \quad \forall t_{\hat{o}}, t \in \mathbb{R}_{>0}
$$

Metric. From the above hypotheses, a radially inhomogeneous metric $g$ is obtained with the same local Ricci curvature as the flat FRW metric [7]. The nonzero elements in comoving polar coordinates $\left(t, r^{\prime}, \theta, \phi\right)$ are $g_{00}=2 k^{-1}(b-1)+1, \quad g_{r^{\prime} r^{\prime}}=-a^{2} / b^{2}, \quad g_{0 r^{\prime}}=-a r^{\prime} / t_{\hat{o}} b$, $g_{\theta \theta}=-a^{2} r^{\prime 2}$ and $g_{\phi \phi}=-a^{2} r^{\prime 2} \sin ^{2} \theta$; where $k^{-1}:=1-$ $\beta_{ \pm}^{2}$ is the spatial curvature, $a(t):=t / t_{\hat{o}}$ is the scale factor and $b\left(r^{\prime}\right)^{2}:=1-k r^{\prime 2} / t_{\hat{o}}^{2}$ is an auxiliary function. The diagonal version of the hyperconical metric is given by the coordinate change $t^{\prime}:=t \sqrt{2 k^{-1}(b-1)+1}$, which is equivalent to selecting $g_{00}^{\prime}=1, g_{0 r}^{\prime}=0$.

Hubble parameter. For the hyperconical model, the scale factor $a$ is initially defined as linear by hypothesis, and thereby it does not depend on the matter-energy contents. The Hubble parameter is derived using the diagonal coordinates as $H_{\text {hyp }}:=a^{-1} \partial a / \partial t^{\prime}$, i.e., 


$$
H_{\mathrm{hyp}}=\frac{1}{t^{\prime}}=\frac{1}{t_{\hat{o}}} \frac{a_{\hat{o}}}{a}=\frac{1+z}{t_{\hat{o}}}
$$

Comoving distance. In the hyperconical model, two comoving measures are distinghished, the radial coordinate $r_{\text {hyp }}^{\prime}$ of the null geodesic under the extrinsic view and the physical (proper) radial distance $\hat{r}_{\text {hyp }}^{\prime}$ obtained by projection to the intrinsic view of the hyperconical universe. That is

$$
\begin{gathered}
r_{\text {hyp }}^{\prime}(z)=\frac{1}{H_{o}} \xi_{k}^{-1}(\ln (1+z)) \\
\hat{r}_{\text {hyp }}^{\prime}(z):=f_{\hat{r}}^{\alpha}\left(t_{\hat{o}}, r_{\text {hyp }}^{\prime}\right)
\end{gathered}
$$

where $f_{\hat{r}}^{\alpha}$ is a projection map (detailed in Sec. III) and the function $\xi_{k}$, obtained from the null geodesic curve under the diagonal hyperconical metric, is given by

$$
\xi_{k}\left(\frac{r^{\prime}}{t_{\hat{o}}}\right):=\int_{0}^{r^{\prime}} \frac{\sqrt{1-k^{-1}(1-b)^{2}}}{b\left(2 k^{-1}(b-1)+1\right)} \frac{d r^{\prime}}{t_{\hat{o}}}
$$

where $b=b\left(r^{\prime}\right):=\sqrt{1-k r^{\prime 2} / t_{\hat{o}}^{2}}$.

\section{CHOICE OF PROJECTION}

The deformation $\mathcal{T}_{t}: \mathrm{H}_{\beta \pm}^{4} \rightarrow \mathbb{R}_{ \pm}^{5}$ leads to a differential line that provides the metric $g$, but the output of this transformation is in $\mathbb{R}_{ \pm}^{5}$ and still has the unobserved spatial $u$-coordinate. Therefore a projection map $f^{\alpha}: \mathbb{R}_{ \pm}^{5} \rightarrow \mathbb{R}_{g}^{1,3}$ is required to remove $u$, satisfying $f=f^{\alpha} \circ \mathcal{T}_{t}$, i.e.,

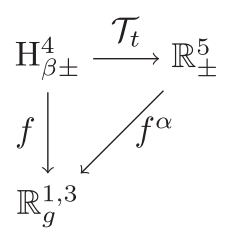

In this work, a family of projection maps $\left\{f^{\alpha}\right\}_{\alpha}$ is tested. Each map is applied to comoving coordinates $\left(t, \vec{r}^{\prime}, u^{\prime}\right)$ as:

$f^{\alpha}:\left(t, \vec{r}^{\prime}, u\right) \rightarrow\left(f_{\hat{t}}^{\alpha}\left(t, \vec{r}^{\prime}\right), f_{\hat{r}}^{\alpha}\left(t, \vec{r}^{\prime}\right)\right)=:\left(\hat{t}, \overrightarrow{\hat{r}}^{\prime}\right) \in \mathbb{R}_{g}^{1,3}$

and it should satisfy several requirements:

(1) Projection type. To preserve the local measurement of proper distances, it must be an azimuthal and locally conformal projection; that is, $f_{\hat{t}}^{\alpha}(t, \overrightarrow{0}) \equiv t$, $f_{\hat{r}}^{\alpha}(t, \overrightarrow{0}) \equiv 0$ and $f_{\hat{r}}^{\alpha}(t, \vec{\epsilon}) \approx \vec{\epsilon}$ for $|\vec{\epsilon}| \ll t$. Therefore, it is spatially isotropic $f_{\hat{r}}^{\alpha}\left(t, \vec{r}^{\prime}\right)=f_{\hat{r}}^{\alpha}\left(t, r^{\prime}\right) \vec{r}^{\prime} / r^{\prime}$ for all $\vec{r}^{\prime}$ such as $r^{\prime}:=\left|\vec{r}^{\prime}\right|>0$.

(2) False boundary. As $\mathcal{T}_{t}$ is a local projection (done by an observer), it produces an apparent boundary at a certain distance. Particularly, the function $\xi_{k}\left(r^{\prime} / t_{\hat{o}}\right)$ is only real in $r^{\prime} \in\left[0, r_{k}^{\prime}\right)$ where

$$
r_{k}^{\prime}:=\frac{t_{\hat{o}}}{2} \sqrt{4-k}
$$

is the boundary of $\mathcal{T}_{t}\left(\mathrm{H}_{\beta \pm}^{4}\right) \ni r^{\prime}$, where $\xi_{k}$ diverges. The existence of this limit leads to a divergence in the first derivative of the projection, i.e., $d f_{\hat{r}}^{\alpha}\left(t, r^{\prime}\right) /\left.d r\right|_{r^{\prime}=r_{k}^{\prime}}=\infty$.

(3) Corrected boundary. To find the correction of the map $f^{\alpha}$, it must be taken into account that the intrinsic measurement of $\left|\vec{r}^{\prime}\right|$ in $\mathbb{R}_{g}^{1,3}$ is limited by the maximum arc length in $\mathrm{H}_{\beta \pm}^{4}$, that is $\pi \nu t_{\hat{o}}=\max \left(\gamma^{\prime} \nu t_{\hat{o}}\right)=\max \left(\left|\overrightarrow{\hat{r}}^{\prime}\right|\right)=f_{\hat{r}}^{\alpha}\left(t_{\hat{o}}, r_{k}^{\prime}\right)$.

Unfortunately, the possible projection map $f_{\hat{r}}^{\alpha}$ that satisfies conditions (1) and (2) s not unique. For instance, we tried a distorted stereographic projection

$$
\begin{gathered}
{ }_{a} f_{\hat{r}}^{\alpha}\left(t_{\hat{o}}, r_{k}^{\prime}\right) \sim \nu t_{\hat{o}} \gamma^{\prime} \Delta^{\alpha}\left(\gamma^{\prime} / \gamma_{k}^{\prime}\right) \\
\Delta^{\alpha}(x):=\frac{1}{(1-x)^{\alpha}}
\end{gathered}
$$

where $\quad \gamma^{\prime}:=\gamma^{\prime}\left(r^{\prime}\right)=\sin ^{-1}\left(r^{\prime} / \nu t_{\hat{o}}\right), \quad \gamma_{k}^{\prime}:=\gamma^{\prime}\left(r_{k}^{\prime}\right) \quad$ and $\Delta^{\alpha}:[0,1) \rightarrow[0, \infty)$ is a stereographic projector radially distorted by the parameter $\alpha \in \mathbb{R}_{\geq 0}$ and applied to a normalized height $x \in[0,1)$. For an observer, the intrinsic measurement of the distances (including the height) is locally given by the arc length $\left(\nu t_{\hat{o}} \gamma^{\prime}\right)$ in $\mathrm{H}_{\beta \pm}^{4}$.

Adding the condition (3), Eq. (17) is only valid for a small region $\left(\gamma^{\prime} \ll 1\right)$ because it diverges when $\gamma^{\prime} \rightarrow \gamma_{k}^{\prime}$, and then it requires eliminating this divergence. For example, we tried the inverse stereographic projection

$$
{ }_{b} f_{\hat{r}}^{\alpha}\left(t_{\hat{o}}, r^{\prime}\right) \sim 2 \nu t_{\hat{o}} \tan ^{-1} \frac{\gamma^{\prime} \Delta^{\alpha}\left(\gamma^{\prime} / \gamma_{k}^{\prime}\right)}{2}
$$

That is, the projection $f_{\hat{r}}^{\alpha}$ remains one-parametric $(\alpha)$. For the temporal projection, it is supposed that $\hat{t}=f_{\hat{t}}^{\alpha}(t, \vec{r}) \approx t$.

\section{THEORETICAL COMPATIBILITY}

\section{A. Equivalent proper distances}

The $\Lambda \mathrm{CDM}$ and hyperconical models can be respectively interpreted as the intrinsic and extrinsic views of a same local universe, i.e., when distance approaches to zero. This local compatibility between the flat FRW metric and the hiperspherical extrinsic metric (hyperconical universe) leads to an equivalence of the proper or comoving distance obtained according both models when only the intrinsic view is used. Specifically,

$$
\int_{0}^{z} \frac{d z}{H_{\Lambda}(z)}=: \hat{r}_{\Lambda}^{\prime}(z) \approx \hat{r}_{\text {hyp }}^{\prime}(z)=: \int_{0}^{z} \frac{d z}{\hat{H}(z)}
$$


for $z \ll 1$, with the corresponding curvatures $(K=0$ for $\Lambda \mathrm{CDM}$ and $k \equiv 1$ for the hyperconical model). In other words, the goal is to find the projection map $f_{\hat{r}}^{\alpha}$ that satisfies a second order (regional) equivalence between an apparent flat Hubble parameter $\hat{H}(z)$ (derived from the projected hyperconical universe) and the measured flat Hubble parameter (according to the $\Lambda \mathrm{CDM}$ model). To analyze the theoretical regional compatibility between both theories, the function $\hat{H}(z)$ is obtained and compared with $H_{\Lambda}$. From the right side of the Eq. (20) and considering Eqs. (12) and (13) it is easy to find that

$$
\hat{H}(z)=\left(\frac{d}{d z^{\prime}} \circ f_{\hat{r}}^{\alpha} \circ \xi_{k}^{-1} \circ \int_{0}^{z^{\prime}} d z \frac{1}{H_{\text {hyp }}}\right)^{-1}(z)
$$

where it is taken as $t_{\hat{o}} \equiv 1$ and thereby $H_{\text {hyp }}(z)=1+z$. The function $\xi_{k}$ is that provided by Eq. (14) and the projection map $f_{\hat{r}}^{\alpha}$ is supposed equal to either ${ }_{a} f_{\hat{r}}^{\alpha}$ [Eq. (17)] or ${ }_{b} f_{\hat{r}}^{\alpha}$ [Eq. (19)]. To distinguish both cases, the related Hubble parameters are denoted as ${ }_{a} \hat{H}(z)$ and ${ }_{b} \hat{H}(z)$.

\section{B. Second order compatibility analysis}

Because the standard $\Lambda$ CDM model has shown good results even for high redshifts, the theoretical compatibility of a hypothetical projection map [Eq. (19)] should be considered not only at the first order. Expanding the theoretical expression of $H_{\Lambda}$ [Eq. (1)] in terms of Taylor series up to second order, $H_{\Lambda}^{(2)}$, it is found that

$$
\begin{aligned}
H_{\Lambda}^{(2)}= & \sqrt{\Omega_{r}+\Omega_{m}+\Omega_{\Lambda}}+\frac{4 \Omega_{r}+3 \Omega_{m}}{\sqrt{\Omega_{r}+\Omega_{m}+\Omega_{\Lambda}}} \frac{z}{2} \\
& +\frac{8 \Omega_{r}^{2}+\left(24 \Omega_{\Lambda}+12 \Omega_{m}\right) \Omega_{r}+12 \Omega_{m} \Omega_{\Lambda}+3 \Omega_{m}^{2}}{\left(\Omega_{r}+\Omega_{m}+\Omega_{\Lambda}\right)^{3 / 2}} \frac{z^{2}}{8}
\end{aligned}
$$

The Hubble parameter derived from the projected hyperconical universe [Eq. (21)], expanded up to second order provides:

$$
\begin{aligned}
\hat{H}^{(2)}= & 1+\frac{\gamma_{k}-2 \alpha \sqrt{k}}{\gamma_{k}} z \\
& +\frac{5 \alpha^{2} k-2 \alpha \sqrt{k} \gamma_{k}-3 \alpha k+m \gamma_{k}^{2}}{2 \gamma_{k}^{2}} z^{2}
\end{aligned}
$$

where either $m=2$ (for $f_{\hat{r}}^{\alpha}={ }_{a} f_{\hat{r}}^{\alpha}$ ) or $m=5 / 2$ (for $f_{\hat{r}}^{\alpha}={ }_{b} f_{\hat{r}}^{\alpha}$ ), with $\alpha$ assumed as approximately constant at second order.

Therefore, the equivalence expressed in the Eq. (20) leads to three equations corresponding to the zero, first, and second orders of $H_{\Lambda}^{(2)}$ and $\hat{H}^{(2)}$. The zeroth order corresponds to the trivial flat FRW universe $\Omega_{r}+\Omega_{m}+\Omega_{\Lambda}=1$. The first order is dependent on the family shape parameter $\alpha$. Even taking the normalization $k \equiv 1$ and assuming that $0 \approx \Omega_{r} \ll 1$, there are still three unknowns $\left(\Omega_{m}, \Omega_{\Lambda}, \alpha\right)$, with two equations. Thus, the second order equality is required to solve the system $H_{\Lambda}^{(2)}=\hat{H}^{(2)}$.

An important point for the reader is that the corresponding system up to third order, $H_{\Lambda}^{(3)}=\hat{H}^{(3)}$, is incompatible with a constant $\alpha$ and $\Omega_{r} \approx 0$. Thereby, the Eqs. (17) and (19) should be considered just as second order approximations with constant $\alpha$.

\section{Explicit second order solution}

The explicit solution of the second order system $H_{\Lambda}^{(2)}=\hat{H}^{(2)}$, given by Eqs. (22) and (23), is

$$
\begin{gathered}
\Omega_{\Lambda}=\frac{3 \alpha^{2} k+2 \alpha \sqrt{k} \gamma_{k}-\alpha k}{2 \gamma_{k}^{2}}+\omega_{\Lambda} \\
\Omega_{m}=\frac{2 \alpha k(1-3 \alpha)}{\gamma_{k}^{2}}+\omega_{m} \\
\Omega_{r}=\frac{9 \alpha^{2} k-2 \alpha \sqrt{k} \gamma_{k}-3 \alpha k}{2 \gamma_{k}^{2}}+\omega_{r}
\end{gathered}
$$

where $\omega_{\Lambda}=1 / 2$ or $(6+k) / 12, \omega_{m}=0$ or $-k / 3, \omega_{r}=$ $1 / 2$ or $(2+k) / 4$ depending on whether ${ }_{a} \hat{H}$ or ${ }_{b} \hat{H}$ is used, as well $\Omega_{\Lambda}+\Omega_{m}+\Omega_{r}=1$.

Considering $k \equiv 1$ and $\Omega_{r} \approx 0\left(\Omega_{r}=9.0 \pm 0.5 \times 10^{-5}\right)$, the numerical solutions correspond to two complex conjugate sets:

$$
\begin{aligned}
\alpha & =0.2830219501(1) \pm c_{\alpha} i \\
\Omega_{\Lambda} & =0.6937181(2) \pm c_{\omega} i \\
\Omega_{m} & =0.306192(6) \mp c_{\omega} i
\end{aligned}
$$

where $\quad c_{\alpha}=0.204263(4) \quad$ or $\quad 0.320386(2), \quad c_{\omega}=$ $0.260076(4)$, or 0.407928 (3) depending on whether ${ }_{a} \hat{H}$ or ${ }_{b} \hat{H}$ is used, and the value in parenthesis is the interval error of last significant figure. Note that the real part of $\Omega_{\Lambda}$ and $\Omega_{m}$ are compatible with the observations updated by the Planck Mission $\left(\Omega_{\Lambda}=0.6911 \pm 0.0062\right.$ and $\Omega_{m}=0.3089 \pm 0.0062$, [11]). If other positive values of $k$ are considered $(0<k<1)$, the real values predicted for the parameters remain about $\Omega_{\Lambda}=0.699 \pm 0.005, \Omega_{m}=$ $0.3001 \pm 0.005$ and $\alpha=0.280 \pm 0.003$.

Proposition.-Since $f_{a} f_{\hat{r}}^{\alpha}$ and ${ }_{b} f_{\hat{r}}^{\alpha} \in C^{\infty}\left[0, r_{k}^{\prime}\right)$ lead to the same real part of $\Omega_{\Lambda}$ and $\Omega_{m}$, there exist smooth $n$ parametric $(\lambda)$ transformations $Q_{\lambda}:{ }_{a} f_{\hat{r}}^{\alpha} \rightarrow{ }_{b} f_{\hat{r}}^{\alpha}$ such as, for only one configuration of $\lambda$ in each transformation, the imaginary part of these cosmological parameters is zero, i.e., $c_{\omega}=0$.

To prove the existence of this unique configuration, it is enough to find the (unique) curves ${ }_{a} \alpha\left(\gamma^{\prime}\right)$ and ${ }_{b} \alpha\left(\gamma^{\prime}\right)$ that, 




FIG. 1. Theoretical compatibility between the standard model and the projected hyperconical universe: (a) Solution curve ${ }_{a} \alpha(z)$ found for ${ }_{a} \hat{H}=H_{\Lambda}$, (b) Solution curve ${ }_{b} \alpha(z)$ found for ${ }_{b} \hat{H}=H_{\Lambda}$.

respectively, solve $c_{\omega}=0$ for ${ }_{a} \hat{H}(z)$ and ${ }_{b} \hat{H}(z)$. Considering the real part of Eq. (27) for $H_{\Lambda}$ and the solution curves ${ }_{a} \alpha\left(\gamma^{\prime}\right)$ and ${ }_{b} \alpha\left(\gamma^{\prime}\right)$, it is obtained that ${ }_{a} \hat{H}(z)={ }_{b} \hat{H}(z)=H_{\Lambda}(z) \quad$ (Fig. 1), i.e., $\quad{ }_{a} f_{\hat{r}}^{a^{\alpha}}={ }_{b} f_{\hat{r}}^{b^{\alpha}}$. Trivially, there exist parametric maps $q_{a}\left(\lambda_{a}\right):{ }_{a} f_{\hat{r}}^{\alpha} \rightarrow$ ${ }_{a} f_{\hat{r}}^{\alpha}$ and $q_{b}^{-1}\left(\lambda_{b}\right):{ }_{b} f_{\hat{r}}^{b^{\alpha}} \rightarrow{ }_{b} f_{\hat{r}}^{\alpha}$. Therefore, one of the possible transformations is $Q_{\lambda}=Q_{\left(\lambda_{a}, \lambda_{b}\right)}:=q_{b}^{-1} \circ q_{a}$ and its unique configuration that solves $c_{\omega}=0$ is $\lambda=\left(\lambda_{a}, 0\right)$.

\section{Agreement with observations}

Empirical values of $\alpha$ were obtained according to 580 Type Ia supernovae ( $\mathrm{SNe}$ Ia) data, collected from the Supernova Cosmology Project (SCP) Union2.1 database ( $z$ from 0.015 to 1.414 ) [12,13]. Theoretical distance modulus was fitted for the projected hyperconical universe [Eq. (13)] using ${ }_{a} f_{\hat{r}}^{\alpha}$ and ${ }_{b} f_{\hat{r}}^{\alpha}$. For $k \equiv 1$ and considering constant the parameter $\alpha$, the best fit was $\alpha=0.30 \pm 0.01$ $\left(\chi_{0}^{2}=562\right)$ for ${ }_{a} f_{\hat{r}}^{\alpha}$ and $\alpha=0.36 \pm 0.02\left(\chi_{0}^{2}=562\right)$ for ${ }_{b} f_{\hat{r}}^{\alpha}$. Within two sigma interval, they are compatible with predicted averages ${ }_{a} \bar{\alpha}=0.290 \pm 0.005$ and ${ }_{b} \bar{\alpha}=$ $0.325 \pm 0.005$, estimated for the considered redshift values $(z \leq 1.414)$.

Finally, the projection ${ }_{b} f_{\hat{r}^{\prime}}^{\alpha}$ can be fitted to obtain the same multipole value for the first CMB peak [Eq. (7)] than the standard model (assuming the empirical baryon-tophoton ratio for both models). The distortion parameter that satisfies this equality is $\alpha=0.320 \pm 0.005$. Therefore, the new model could be compatible with both local and global scales for a distortion of $\alpha=0.325 \pm 0.005$.

\section{E. Summary and conclusions}

From the hypothesis of a linearly expanding hypersphere (hypercone), it is obtained an inhomogeneous metric that approaches to an effective flat FRW metric at local scale. This is equivalent to a hypothetical compensation of the matter effect (deceleration) by a dark energy effect (acceleration), at least locally. Consistently, there is a mathematically compatibility between the expansion explained under the standard model and under the view of the hyperconical model.

The (intrinsic) proper distance obtained from the standard model could be interpreted as a locally conformal projection of the comoving radial coordinate of the (extrinsic) hyperconical universe. This projection causes a distortion that would provide the origin of the cosmological constant. Particularly, imposing equality in second order, a dark energy density is obtained about $\Omega_{\Lambda} \approx 0.7$. Moreover, it is found a consistent projection $\left({ }_{b} f_{\hat{r}^{\prime}}^{\alpha}\right)$, whose predicted distortion parameter for low redshifts $(\alpha=0.325 \pm 0.005$, $z \leq 1.414)$ is compatible with its empirical value ( $\alpha=0.36 \pm 0.02$, fitted to the SNe Ia observations), and very similar to the theoretical value $(\alpha=0.320 \pm 0.005)$ obtained at high redshifts to explain the first CMB acoustic peak. However, the projection proposed in this paper is just an example and other possibilities could produce better results.

\section{ACKNOWLEDGMENTS}

The encouragement and helpful comments received from Prof. R. Campoamor-Stursberg are gratefully acknowledged.
[1] J. B. Jimenez, R. Lazkoz, and A. L. Maroto, Phys. Rev. D 80, 023004 (2009).

[2] P. D. Mannheim, Prog. Part. Nucl. Phys. 56, 340 (2006).
[3] P. D. Mannheim, Imperial College London Report No. PASCOS-07, 2007; arXiv:0707.2283.

[4] M. D. Maia, E. M. Monte, J. M. F Maia, and J. S. Alcaniz, Classical Quantum Gravity 22, 1623 (2005). 
[5] F. Melia, Mon. Not. R. Astron. Soc. 382, 1917 (2007).

[6] A. Benoit-Lévy and G. Chardin, Astron. Astrophys. 537, A78 (2012).

[7] R. Monjo, Phys. Rev. D 96, 103505 (2017).

[8] T. Buchert, Gen. Relativ. Gravit. 40, 467 (2008).

[9] W. Hu and M. White, Astrophys. J. 471, 30 (1996).
[10] Q.-G. Huang, K. Wanga, and S. Wanga, J. Cosmol. Astropart. Phys. 12 (2015) 022

[11] Planck Collaboration, Astron. Astrophys. 594, A8 (2015).

[12] D. R. Kowalski et al., Astron. J. 686, 749 (2008).

[13] N. Suzuki et al., Astrophys. J. 746, 85 (2012). 